\title{
Identification of Metabolic Biomarkers for Personalized Nutrition
}

\author{
Jing X. Kang \\ Massachusetts General Hospital and Harvard Medical School, Boston, Mass., USA
}

Now more than ever, we have begun to appreciate the roles of individual physical and genetic variation in health and medicine. A great deal of research attention has recently been devoted toward personalized medicine, in which technologies like medical genetics are used to determine information such as how a patient is likely to respond to a treatment or the optimal dose of a drug. While drug therapy will undoubtedly advance as a result of this new focus, it is critical that we not overlook the importance of personalized nutrition in disease management and health promotion. Indeed, personalized nutrition may be even more critical than personalized medicine given that dietary intake is a fundamental part of everyday life that affects our health.

The fields of nutrigenetics and nutrigenomics present uniquely powerful sources of information for implementing personalized nutrition. Nutrigenetics specifies how individual genetic variance impacts response to nutrients such that an individual can avoid negative metabolic consequences by choosing appropriate foods. One classic example is lactose intolerance, in which one lacks the lactase persistence allele and therefore cannot digest lactose [1]. Thus, this person's health would benefit from avoiding foods containing lactose. On the other hand, nutrigenomics explores how nutrients affect gene expression, so that ingesting certain nutrients according to an individual's health condition can normalize their diseaserelated gene expression. For example, increased systemic inflammation is linked to an upregulation of inflammatory cytokines (e.g. TNF- $\alpha$, IL-1, IL-6), which can in turn be suppressed by some nutrients (e.g. omega-3 fatty acids [2]). The key element linking these 'sister' fields is the concept that nutrients interact with an individual's genetic profile to impact their health. Thus, as each individual has their own unique genetic profile, this knowledge is essential for developing personalized nutrition.

The critical prerequisite for personalized nutrition is the ability to precisely detect and interpret an individual's metabolic and health status by way of metabolic biomarkers. These 
biomarkers are primarily concerned with the molecular signatures related to pathways of metabolism, which include the genes or proteins responsible for nutrient metabolism as well as the metabolites derived from a metabolic pathway. For example, biomarkers for diabetes might include dysregulated insulin expression and elevated levels of blood glucose, both of which reflect disordered glucose metabolism. Therefore, it is important for the development of personalized nutrition to identify metabolic biomarkers, especially those that are: (a) linked to a pathological or disease condition; (b) easily measurable (e.g. by plasma or urine samples), and (c) modifiable by nutrient intake. However, such biomarkers are currently still rare, and great efforts should be directed toward this endeavor.

An integrated approach should be emphasized in the identification of metabolic biomarkers. Currently, biomarker identification efforts often focus on a singular molecular species, particularly mRNA, proteins, or metabolites. While each of these measurements shows one view of a particular pathway, the integration of these methods can provide a full and dynamic perspective for the interaction between genes and nutrients. Alterations in metabolic pathways will usually involve changes in gene expression levels as well as metabolite content. These gene changes can be detected by gene expression analysis, such as next-generation sequencing or proteomics [3], while metabolite changes can be detected by metabolomic analysis [4]. For example, next-generation sequencing identifies gene alterations in a particular pathway, such as increased COX-2 expression in eicosanoid formation. On the other hand, metabolomic analysis indicates the levels of corresponding metabolites in the pathway, such as increased content of prostaglandin E2 (PGE2, a COX-2 metabolite). By putting together this information, we can confirm that eicosanoid production is augmented. As shown, the combination of these technologies that allow for detecting changes in both genes and nutrients can provide us with a comprehensive picture of metabolic pathways and the networks between key elements, while reducing confounding factors and increasing accuracy in the identification of metabolic biomarkers.

To establish personalized nutrition, a great deal of work remains to be done. By harnessing new technologies in integrative ways, it is my hope that we can quickly and accurately identify an individual's nutritional needs, and use this knowledge as a powerful adjunct to current health interventions. To this end, it is now a priority for researchers to develop new metabolic biomarkers that are both easily detected and responsive to nutritional interventions. Research in nutrigenetics and nutrigenomics will be instrumental in achieving this goal.

\section{References}

1 Swallow DM: Genetics of lactase persistence and lactose intolerance. Annu Rev Genet 2003;37:197-219.

- Kang JX, Weylandt KH: Modulation of inflammatory cytokines by omega-3 fatty acids. Subcell Biochem 2008;49: 133-143.

3 Mortazavi A, Williams BA, McCue K, Schaeffer L, Wold B: Mapping and quantifying mammalian transcriptomes by RNA-Seq. Nat Methods 2008;5:621-628.

4 Patti GJ, Yanes O, Siuzdak G: Metabolomics: the apogee of the omics trilogy. Nat Rev 2012;13:263-269. 